\title{
Kumi Yasuda \\ On the asymptotic growth rate of the sum of $p$-adic-valued independent identically distributed random variables
}

Received: 27 December 2013 / Accepted: 25 May 2014 / Published online: 19 June 2014

(C) The Author(s) 2014. This article is published with open access at Springerlink.com

\begin{abstract}
The $p$-adic semistable laws are characterized as weak limits of scaled summands of $p$-adic-valued, rotation-symmetric, independent, and identically distributed random variables whose tail probability satisfies some condition. In this article it is verified such scaled sums do not converge in probability, and some more precise estimates, corresponding to the law of iterated logarithm in the real-valued setting, are given to the asymptotic growth rate of the sum. The critical scaling order is explicitly given, over which the scaled sum almost surely converges to 0 . On the other hand, under the critical order, the limit superior of the $p$-adic norm of the scaled sum diverges almost surely. Furthermore it is shown that, at the critical order, a crucial change of the asymptotic behavior of the scaled sum occurs according to the decay of the tail probability of the random variables. In this situation, the critical value for the order of the tail probability is also found.
\end{abstract}

Mathematics Subject Classification 60G50 - 11F85 - 60F05

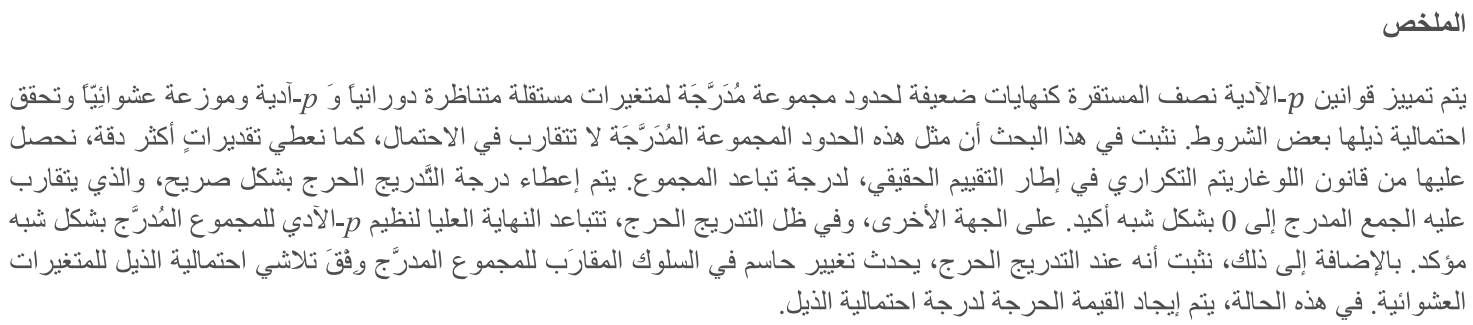

\section{Introduction and main theorems}

Limit theorems for sums of real-valued independent random variables are one of the core subjects in probability theory. It is known that weak limits of sequences of normalized sums of independent and identically distributed (i.i.d.) random variables form the whole set of stable distributions [1,3]. The central limit theorem assures that, under some additional conditions, the limits are the standard normal distribution. However, they are not convergent in stronger senses, such as almost surely or in probability, and in this situation the law of the iterated logarithm gives a more precise estimates for the growth rate of the sums [2,3].

Concerning with the $p$-adic-valued random variables, limit distributions of the (scaled) sums of rotationsymmetric i.i.d. $\xi_{i}(i=1,2, \ldots)$ are discussed in [4]. Here, a rotation-symmetric random variable is such $\xi$

K. Yasuda $(\varangle)$

Faculty of Business and Commerce, Keio University, 4-1-1 Hiyoshi, Kohoku-ku, Yokohama 223-8521, Japan

E-mail:kumi@a6.keio.jp 
that $P(\xi \in X)=P(\xi \in u X)$ for any measurable subset $X$ of the $p$-adic field and any unit $u$ in the ring of the $p$-adic integers. In case the $\xi_{i}$ are bounded and non-degenerate, the ultra-metric property of the $p$-adic norm brings a different situation from the Euclidean case; the distribution of the sum, without any scaling, converges to the normalized Haar measure on the minimum disc centered at the origin that contains the support of $\xi_{i}$. On the other hand, if we consider unbounded $\xi_{i}$, " $p$-adic semistable laws" appear as the weak limit distributions of the scaled sums. In this article, we will show the convergence to the $p$-adic semistable laws does not follow in stronger senses, and determine the precise growth rate of the sum.

Throughout this article, we fix a prime integer $p$. For a non-zero integer $z$, the $p$-adic norm of $z$ is defined by $|z|_{p}=p^{-m}$, where $m$ is the maximum integer such that $p^{m}$ divides $z$. The $p$-adic norm is naturally extended to rational numbers, if we define $|0|_{p}=0$ and $\left|\frac{z}{z^{\prime}}\right|_{p}=\frac{|z|_{p}}{\left|z^{\prime}\right|_{p}}$ for integers $z$ and $z^{\prime} \neq 0$. The field of $p$-adic numbers, denoted by $\mathbf{Q}_{p}$, is the topological closure of $\mathbf{Q}$ with respect to the $p$-adic norm. The non-zero elements of $\mathbf{Q}_{p}$ are identified with formal series $x=\sum_{i=m}^{\infty} a_{i} p^{i}$ with $m \in \mathbf{Z}, a_{i} \in\{0,1,2, \ldots, p-1\}, a_{m} \neq 0$, and they are normed by $|x|_{p}=p^{-m}$. The $p$-adic norm satisfies the ultra-metric inequality : $\left|x+x^{\prime}\right|_{p} \leq \max \left\{|x|_{p},\left|x^{\prime}\right|_{p}\right\}$, and this characteristic property is frequently used for some estimations in the followings.

A character on $\mathbf{Q}_{p}$ is a continuous homomorphism on the additive group of $p$-adic numbers to the multiplicative group of complex numbers with absolute value 1 . For $p$-adic numbers $x$, let us define

$$
\varphi_{1}(x):= \begin{cases}1, & \text { if } \quad x=0 \text { or } x=\sum_{i=m}^{\infty} a_{i} p^{i} \quad \text { with } m \geq 0, \\ \exp \left(2 \pi \sqrt{-1} \sum_{i=m}^{-1} a_{i} p^{i}\right), & \text { if } \quad x=\sum_{i=m}^{\infty} a_{i} p^{i} \quad \text { with } m \leq-1 .\end{cases}
$$

Then $\varphi_{1}$ is a character on $\mathbf{Q}_{p}$, and any character $\varphi$ is of the form $\varphi(x)=\varphi_{y}(x):=\varphi_{1}(y x)$ for some $y \in \mathbf{Q}_{p}$. For a distribution $\mu$ on the field of $p$-adic numbers, its characteristic function is defined on the group of characters by $\hat{\mu}(\varphi):=\int_{\mathbf{Q}_{p}} \varphi(x) \mu(d x)$. The characteristic function is considered to be a function on $\mathbf{Q}_{p}$ through the identification $\varphi_{y} \leftrightarrow y$, and written as $\hat{\mu}(y):=\hat{\mu}\left(\varphi_{y}\right), y \in \mathbf{Q}_{p}$.

Definition 1.1 For $\alpha>0$, a distribution $\mu$ on the field of $p$-adic numbers is called a ( $p$-adic) $\alpha$-semistable law, if its characteristic function is given by

$$
\hat{\mu}(y)=e^{-c|y|_{p}^{\alpha}}, \quad y \in \mathbf{Q}_{p}
$$

for some constant $c>0$.

For $p$-adic-valued i.i.d. $\xi_{i}(i=1,2, \ldots)$, we shall denote their tail probability by $T(s):=P\left(\left|\xi_{i}\right|_{p} \geq s\right)$, $s \geq 0$, and let $S_{n}:=\sum_{i=1}^{n} \xi_{i}$ be their partial sums. The $p$-adic semistable laws are characterized as limit distributions of scaled sums of rotation-symmetric i.i.d. as follows.

Proposition 1.2 ([4] Theorem 2) A distribution $\mu$ on the field of p-adic numbers is semistable, if and only if it is a non-degenerate limit distribution of the scaled sum $p^{n} S_{k(n)}$ for some rotation-symmetric i.i.d. $\xi_{i}(i=$ $1,2, \ldots)$ and some increasing sequence $\{k(n)\}_{n=1,2, \ldots}$ of natural numbers satisfying $\sup _{n} k(n) T\left(p^{n+l}\right)<$ $+\infty$ for any integer $l$.

In what follows, the integer part of a real number $t$ is denoted by $[t]$. For a random variable $X, \mathcal{L}(X)$ denotes the law of $X$, and $\hat{\mathcal{L}}(X)$ its characteristic function. We let $\mu_{\alpha}$ be the $\alpha$-semistable law with the characteristic function $\hat{\mu}_{\alpha}(y)=e^{-|y|_{p}^{\alpha}}$.

Proposition 1.3 ([4] Proposition 4) Suppose the tail probability $T$ of the rotation symmetric i.i.d. $\xi_{i}$ is given by

$$
T\left(p^{n}\right)=p^{-\alpha n} L(n), \quad n \in \mathbf{Z},
$$

for some $\alpha>0$ and some sequence of positive numbers $L(n)$ satisfying $\lim _{n \rightarrow \infty} \frac{L(n+1)}{L(n)}=1$. If we put $N(n):=C_{\alpha} T\left(p^{n}\right)^{-1}, C_{\alpha}:=\frac{p^{2 \alpha}(p-1)}{p^{\alpha+1}-1}$, then the law of the scaled sum $p^{n} S_{[N(n)]}$ converges to $\mu_{\alpha}$ as $n \rightarrow \infty$.

Under the condition of Proposition 1.3, we will determine the growth rate of $\left|S_{[N(n)]}\right|_{p}$. 
Theorem 1.4 Let $\beta>0$, and put $c_{n}=[\beta \log n]$.

(i) If $\beta>\frac{1}{\alpha \log p}$, then $\lim \sup _{n \rightarrow \infty}\left|p^{n+c_{n}} S_{[N(n)]}\right|_{p}=0$, and $p^{n+c_{n}} S_{[N(n)]}$ converges to 0 almost surely.

(ii) If $\beta<\frac{1}{\alpha \log p}$, then $\lim \sup _{n \rightarrow \infty}\left|p^{n+c_{n}} S_{[N(n)]}\right|_{p}=+\infty$ almost surely.

We shall examine next what happens at the critical order $c_{n} \sim \frac{\log n}{\alpha \log p}$. As the following theorem shows, the both cases $\lim \sup _{n \rightarrow \infty}\left|p^{n+c_{n}} S_{[N(n)]}\right|_{p}=0$ and $=+\infty$ may occur according to the order of the convergence $\frac{L(n+1)}{L(n)} \rightarrow 1$.

Theorem 1.5 Let $c_{n}=\left[\frac{\log n}{\alpha \log p}\right]$, and suppose $\frac{L(n+1)}{L(n)}=(\log n)^{-\gamma / \log n}$ for $n \geq 2$ with some $\gamma>0$.

(i) If $\gamma>\alpha \log p$, then $\lim \sup _{n \rightarrow \infty}\left|p^{n+c_{n}} S_{[N(n)]}\right|_{p}=0$, and $p^{n+c_{n}} S_{[N(n)]}$ converges to 0 almost surely.

(ii) If $\gamma \leq \alpha \log p$, then $\lim \sup _{n \rightarrow \infty}\left|p^{n+c_{n}} S_{[N(n)]}\right|_{p}=+\infty$ almost surely.

Proofs to Theorems 1.4 and 1.5 will be given in Sect. 4 .

\section{Convergence of the scaled sum}

In the followings let $\alpha>0,\{L(n)\}$ be a sequence of positive numbers such that $\lim _{n \rightarrow \infty} \frac{L(n+1)}{L(n)}=1$, and $\xi_{i}$ be $p$-adic-valued, rotation symmetric i.i.d. with the tail probability $T\left(p^{n}\right)=p^{-\alpha n} L(n)$. Besides the weak convergence confirmed in Proposition 1.3, let us verify whether the scaled sum $p^{n} S_{[N(n)]}$ converges in any stronger sense.

Proposition 2.1 Under the condition of Proposition 1.3, the scaled sum $p^{n} S_{[N(n)]}$ does not converge almost surely.

Proof We have

$$
\begin{aligned}
p^{2 n} S_{[N(2 n)]}-p^{n} S_{[N(n)]} & =p^{2 n}\left(\sum_{i=1}^{[N(n)]} \xi_{i}+\sum_{i=[N(n)]+1}^{[N(2 n)]} \xi_{i}\right)-p^{n} \sum_{i=1}^{[N(n)]} \xi_{i} \\
& =p^{2 n} \sum_{i=[N(n)]+1}^{[N(2 n)]} \xi_{i}-\left(1-p^{n}\right) p^{n} S_{[N(n)]},
\end{aligned}
$$

and by Proposition 1.3 and $1-p^{n} \rightarrow 1$ in $\mathbf{Q}_{p}$, the law of $\left(1-p^{n}\right) p^{n} S_{[N(n)]}$ converges to $\mu_{\alpha}$. Since the random variables $p^{2 n} \sum_{i=[N(n)]+1}^{[N(2 n)]} \xi_{i}$ and $\left(1-p^{n}\right) p^{n} S_{[N(n)]}$ are independent, comparing the characteristic functions of the both sides of (1) gives

$$
\begin{aligned}
& \left\|\hat{\mathcal{L}}\left(p^{2 n} S_{[N(2 n)]}-p^{n} S_{[N(n)]}\right)(y)\right\| \\
& =\left\|\hat{\mathcal{L}}\left(p^{2 n} \sum_{i=[N(n)]+1}^{[N(2 n)]} \xi_{i}\right)(y)\right\| \cdot\left\|\hat{\mathcal{L}}\left(\left(1-p^{n}\right) p^{n} S_{[N(n)]}\right)(y)\right\| \\
& \quad \leq\left\|\hat{\mathcal{L}}\left(\left(1-p^{n}\right) p^{n} S_{[N(n)]}\right)(y)\right\| \\
& \quad \rightarrow\left\|\hat{\mu_{\alpha}}(y)\right\|=e^{-|y|_{p}^{\alpha}}, \quad n \rightarrow \infty,
\end{aligned}
$$

for each $p$-adic number $y$. If we suppose $p^{n} S_{[N(n)]}$ is convergent almost surely, then $p^{2 n} S_{[N(2 n)]}-p^{n} S_{[N(n)]}$ converges to 0 almost surely. Then the left-hand side of (2) should tend to 1 , which is a contradiction.

Furthermore, we can show the convergence in Proposition 1.3 is not even in probability.

Proposition 2.2 Under the condition of Proposition 1.3, the scaled sum $p^{n} S_{[N(n)]}$ does not converge in probability. 
Proof Take a random variable $X_{\alpha}$ whose law is $\mu_{\alpha}$. If we suppose $p^{n} S_{[N(n)]}$ converges to $X_{\alpha}$ in probability, then for any $\varepsilon>0$ we have $P\left(\left|p^{n} S_{[N(n)]}-X_{\alpha}\right|_{p}>\varepsilon\right) \rightarrow 0$ as $n \rightarrow \infty$. The ultra-metric property implies $\left|p^{2 n} S_{[N(2 n)]}-p^{n} S_{[N(n)]}\right|_{p} \leq \max \left\{\left|p^{2 n} S_{[N(2 n)]}-X_{\alpha}\right|_{p},\left|p^{n} S_{[N(n)]}-X_{\alpha}\right|_{p}\right\}$, and therefore

$$
\begin{aligned}
& P\left(\left|p^{2 n} S_{[N(2 n)]}-p^{n} S_{[N(n)]}\right|_{p}>\varepsilon\right) \\
& \quad \leq P\left(\left|p^{2 n} S_{[N(2 n)]}-X_{\alpha}\right|_{p}>\varepsilon\right)+P\left(\left|p^{n} S_{[N(n)]}-X_{\alpha}\right|_{p}>\varepsilon\right) \rightarrow 0 .
\end{aligned}
$$

This shows that $p^{2 n} S_{[N(2 n)]}-p^{n} S_{[N(n)]}$ converges to 0 in probability, and hence in law. Then we have $\left\|\hat{\mathcal{L}}\left(p^{2 n} S_{[N(2 n)]}-p^{n} S_{[N(n)]}\right)(y)\right\| \rightarrow 1$ for any $y \in \mathbf{Q}_{p}$, which contradicts (2) again.

\section{Limit superior of the norm of the scaled sum}

Next let us study the limit superior of $\left|p^{n} S_{[N(n)]}\right|_{p}$. For an integer $m$ and natural numbers $k>l$, put $A_{m}(k, l):=$ $P\left(\left|S_{k}-S_{l}\right|_{p}<p^{m}\right)$.

\section{Lemma 3.1}

$$
A_{m}(k, l) \leq \frac{1}{2}\left(1-2 T\left(p^{m}\right)\right)^{k-l}+\frac{1}{2} .
$$

Proof Since $S_{k}-S_{l}=S_{k-1}-S_{l}+\xi_{k}$, the ultra-metric property implies that, $\left|S_{k}-S_{l}\right|_{p}<p^{m}$ holds only if either $\left|S_{k-1}-S_{l}\right|_{p},\left|\xi_{k}\right|_{p}<p^{m}$ or $\left|S_{k-1}-S_{l}\right|_{p},\left|\xi_{k}\right|_{p} \geq p^{m}$. Hence we can see

$$
\begin{aligned}
A_{m}(k, l) & \leq P\left(\left|S_{k-1}-S_{l}\right|_{p}<p^{m},\left|\xi_{k}\right|_{p}<p^{m}\right)+P\left(\left|S_{k-1}-S_{l}\right|_{p} \geq p^{m},\left|\xi_{k}\right|_{p} \geq p^{m}\right) \\
& =A_{m}(k-1, l)\left(1-T\left(p^{m}\right)\right)+\left(1-A_{m}(k-1, l)\right) T\left(p^{m}\right) \\
& =A_{m}(k-1, l)\left(1-2 T\left(p^{m}\right)\right)+T\left(p^{m}\right),
\end{aligned}
$$

since the random variables $S_{k-1}-S_{l}$ and $\xi_{k}$ are independent. Inductively we can derive that

$$
\begin{aligned}
A_{m}(k, l) & \leq A_{m}(l+1, l)\left(1-2 T\left(p^{m}\right)\right)^{k-l-1}+T\left(p^{m}\right) \sum_{j=0}^{k-l-2}\left(1-2 T\left(p^{m}\right)\right)^{j} \\
& =\left(1-T\left(p^{m}\right)\right)\left(1-2 T\left(p^{m}\right)\right)^{k-l-1}+T\left(p^{m}\right) \frac{1-\left(1-2 T\left(p^{m}\right)\right)^{k-l-1}}{1-\left(1-2 T\left(p^{m}\right)\right)} \\
& =\frac{1}{2}\left(1-2 T\left(p^{m}\right)\right)^{k-l}+\frac{1}{2}
\end{aligned}
$$

Proposition 3.2 Under the condition of Proposition 1.3,

$$
\limsup _{n \rightarrow \infty}\left|p^{n} S_{[N(n)]}\right|_{p}=+\infty \text {, a.s. }
$$

Proof Since $T\left(p^{n}\right) \rightarrow 0$ and $\frac{L(n)}{L(n-1)} \rightarrow 1$, we can see

$$
N(n)-N(n-1)=C_{\alpha} T\left(p^{n}\right)^{-1}\left(1-p^{-\alpha} \frac{L(n)}{L(n-1)}\right) \rightarrow+\infty,
$$

as $n \rightarrow \infty$. Then we can assume $[N(n)]>[N(n-1)]$ for large $n$. For natural numbers $n$ and $s$, put $R_{n, s}:=P\left(\left|S_{[N(n)]}-S_{[N(n-1)]}\right|_{p} \geq p^{n+s}\right)$, then according to Lemma 3.1 it follows that

$$
\begin{aligned}
R_{n, s} & =1-A_{n+s}([N(n)],[N(n-1)]) \\
& \geq \frac{1}{2}\left(1-\left(1-2 T\left(p^{n+s}\right)\right)^{[N(n)]-[N(n-1)]}\right) .
\end{aligned}
$$


Here let us put $N=[N(n)]-[N(n-1)]$ and $t=2 T\left(p^{n+s}\right)$, then

$$
\begin{aligned}
N t & \geq(N(n)-N(n-1)-1) t \\
& =2 C_{\alpha} p^{\alpha n} L(n)^{-1}\left(1-p^{-\alpha} \frac{L(n)}{L(n-1)}\right) \cdot p^{-\alpha(n+s)} L(n+s)-t \\
& =2 C_{\alpha} p^{-\alpha s} \frac{L(n+s)}{L(n)}\left(1-p^{-\alpha} \frac{L(n)}{L(n-1)}\right)-t \\
& =2 C_{\alpha} p^{-\alpha s} \frac{L(n+1)}{L(n)} \cdot \frac{L(n+2)}{L(n+1)} \cdots \frac{L(n+s)}{L(n+s-1)}\left(1-p^{-\alpha} \frac{L(n)}{L(n-1)}\right)-t .
\end{aligned}
$$

Since $\frac{L(n)}{L(n-1)} \rightarrow 1$, we can take $M \geq 1$ such that $p^{-\alpha / 2}<\frac{L(n)}{L(n-1)}<p^{\alpha / 2}$ holds for all $n \geq M$. Then the above implies

$$
\begin{aligned}
t & \geq \frac{1}{N+1} \cdot 2 C_{\alpha} p^{-\alpha s}\left(p^{-\alpha / 2}\right)^{s}\left(1-p^{-\alpha} \cdot p^{\alpha / 2}\right) \\
& =\frac{1}{N+1} \cdot 2 C_{\alpha} p^{-3 \alpha s / 2}\left(1-p^{-\alpha / 2}\right),
\end{aligned}
$$

for $n \geq M$. Putting $\eta:=2 C_{\alpha} p^{-3 \alpha s / 2}\left(1-p^{-\alpha / 2}\right)$, (3) reads

$$
R_{n, s} \geq \frac{1}{2}\left(1-\left(1-\frac{\eta}{N+1}\right)^{N}\right)
$$

Since $N \geq N(n)-N(n-1)-1 \rightarrow+\infty$ as $n \rightarrow \infty$, the right-hand side of (4) tends to $\frac{1}{2}\left(1-e^{-\eta}\right)>0$. Hence we obtain $\sum_{n=1}^{\infty} R_{n, s}=+\infty$. Since the events $\left|S_{[N(n)]}-S_{[N(n-1)]}\right|_{p} \geq p^{n+s}$ are independent for $n=1,2, \ldots$, Borel's theorem yields

$$
P\left(\left|S_{[N(n)]}-S_{[N(n-1)]}\right|_{p} \geq p^{n+s} \text { for infinitely many } n\right)=1 .
$$

Let us show (5) implies $P\left(\left|p^{n} S_{[N(n)]}\right|_{p} \geq p^{s}\right.$ for infinitely many $\left.n\right)=1$. Suppose $\left|S_{[N(n)]}(\omega)\right|_{p}<p^{n+s}$ for all large $n$, then by the ultra-metric property

$$
\left|S_{[N(n)]}(\omega)-S_{[N(n-1)]}(\omega)\right|_{p} \leq \max \left\{\left|S_{[N(n)]}(\omega)\right|_{p},\left|S_{[N(n-1)]}(\omega)\right|_{p}\right\}<p^{n+s}
$$

holds for all large $n$. Whereas such an event occurs with probability 0 according to (5), and thus we obtain $P\left(\left|p^{n} S_{[N(n)]}\right|_{p} \geq p^{s}\right.$ for infinitely many $\left.n\right)=1$. Consequently lim $\sup _{n \rightarrow \infty}\left|p^{n} S_{[N(n)]}\right|_{p} \geq p^{s}$ holds with probability 1, and letting $s \rightarrow \infty$, we obtain $\lim \sup _{n \rightarrow \infty}\left|p^{n} S_{[N(n)]}\right|_{p}=+\infty$ almost surely.

In what follows we consider a non-decreasing sequence of non-negative integers $\left\{c_{n}\right\}$, and look into the behavior of lim $\sup _{n \rightarrow \infty}\left|p^{n+c_{n}} S_{[N(n)]}\right|_{p}$. We will see it may converge or diverge according to the sequences $\left\{c_{n}\right\}$ and $\{L(n)\}$. Put $\mathcal{S}:=\sum_{n=1}^{\infty} p^{-\alpha c_{n}} \frac{L\left(n+c_{n}\right)}{L(n)}$.

Lemma 3.3 (i) If $\mathcal{S}<+\infty$, then $\lim \sup _{n \rightarrow \infty}\left|p^{n+c_{n}} S_{[N(n)]}\right|_{p}=0$, and $p^{n+c_{n}} S_{[N(n)]}$ converges to 0 almost surely.

(ii) If $\mathcal{S}=+\infty$, then $\lim \sup _{n \rightarrow \infty}\left|p^{n+c_{n}} S_{[N(n)]}\right|_{p}=+\infty$ almost surely.

Proof In case $c_{n}$ is bounded, we have $\mathcal{S}=+\infty$ and $\lim \sup _{n \rightarrow \infty}\left|p^{n+c_{n}} S_{[N(n)]}\right|_{p}=+\infty$. Indeed, we can take $M \in \mathbf{N}$ such that $\frac{L(n+1)}{L(n)}>\frac{1}{2}$ for any $n \geq M$, by the condition $\frac{L(n+1)}{L(n)} \rightarrow 1$. If we put $C:=\sup _{n} c_{n}$, then we have

$$
\frac{L\left(n+c_{n}\right)}{L(n)}=\frac{L(n+1)}{L(n)} \cdot \frac{L(n+2)}{L(n+1)} \cdots \frac{L\left(n+c_{n}\right)}{L\left(n+c_{n}-1\right)}>\left(\frac{1}{2}\right)^{c_{n}} \geq\left(\frac{1}{2}\right)^{C},
$$


and $p^{-\alpha c_{n}} \geq p^{-\alpha C}$ for $n \geq M$, which yields $\mathcal{S}=+\infty$ clearly. On the other hand, Proposition 3.2 implies

$$
\limsup _{n \rightarrow \infty}\left|p^{n+c_{n}} S_{[N(n)]}\right|_{p} \geq p^{-C} \limsup _{n \rightarrow \infty}\left|p^{n} S_{[N(n)]}\right|_{p}=+\infty
$$

and thus the case where $c_{n}$ is bounded corresponds to the assertion (ii).

We shall assume $c_{n} \rightarrow+\infty$ in the followings.

(i) Fix a natural number $s$, and define $P_{n, s}:=P\left(\max _{1 \leq i \leq[N(n)]}\left|S_{i}\right|_{p} \geq p^{n+c_{n}-s}\right)$ for $n=1,2, \ldots$ By the ultra-metric property, $\max _{1 \leq i \leq[N(n)]}\left|S_{i}\right|_{p} \geq p^{n+c_{n}-s}$ holds if and only if $\max _{1 \leq i \leq[N(n)]}\left|\xi_{i}\right|_{p} \geq p^{n+c_{n}-s}$, and since $\xi_{i}$ are independent, we have

$$
\begin{aligned}
P_{n, s} & =1-P\left(\left|\xi_{i}\right|_{p}<p^{n+c_{n}-s}, i=1,2, \ldots,[N(n)]\right) \\
& =1-\prod_{i=1}^{[N(n)]} P\left(\left|\xi_{i}\right|_{p}<p^{n+c_{n}-s}\right) \\
& =1-\left(1-T\left(p^{n+c_{n}-s}\right)\right)^{[N(n)]} .
\end{aligned}
$$

Here note that for $t>0$ and a natural number $N$,

$$
\begin{aligned}
1- & (1-t)^{N} \\
= & -\sum_{k=1}^{N}\left(\begin{array}{l}
N \\
k
\end{array}\right)(-t)^{k} \\
& =\left\{\begin{array}{c}
N t-\sum_{j=1}^{\frac{N-1}{2}}\left(\left(\begin{array}{c}
N \\
2 j
\end{array}\right) t^{2 j}-\left(\begin{array}{c}
N \\
2 j+1
\end{array}\right) t^{2 j+1}\right), \quad \text { if } \quad N \text { is odd, } \\
N t-\sum_{j=1}^{\frac{N-2}{2}}\left(\left(\begin{array}{c}
N \\
2
\end{array}\right) t^{2 j}-\left(\begin{array}{c}
N \\
2 j+1
\end{array}\right) t^{2 j+1}\right)-t^{N} \\
\quad \leq N t-\sum_{j=1}^{\frac{N-2}{2}}\left(\left(\begin{array}{c}
N \\
2 j
\end{array}\right) t^{2 j}-\left(\begin{array}{c}
N \\
2 j+1
\end{array}\right) t^{2 j+1}\right), \quad \text { if } \quad N \text { is even, }
\end{array}\right.
\end{aligned}
$$

and the each term in these sums is estimated as

$$
\begin{aligned}
& \left(\begin{array}{l}
N \\
2 j
\end{array}\right) t^{2 j}-\left(\begin{array}{c}
N \\
2 j+1
\end{array}\right) t^{2 j+1} \\
& =\frac{N !}{(2 j+1) !(N-2 j) !} t^{2 j}((2 j+1)-(N-2 j) t) \\
& \geq \frac{N !}{(2 j+1) !(N-2 j) !} t^{2 j}(3-N t) .
\end{aligned}
$$

By the assumptions $\frac{L(n+1)}{L(n)} \rightarrow 1$ and $c_{n} \rightarrow+\infty$, there exists $M \geq 1$ such that $p^{-\alpha / 2}<\frac{L(n+1)}{L(n)}<p^{\alpha / 2}$ and $c_{n}>s$ for all $n \geq M$. Let $n \geq M$ and put $N=[N(n)], t=T\left(p^{n+c_{n}-s}\right)$, then

$$
\begin{aligned}
N t & \leq N(n) t \\
& =C_{\alpha} p^{-\alpha\left(c_{n}-s\right)} \frac{L\left(n+c_{n}-s\right)}{L(n)} \\
& =C_{\alpha} p^{-\alpha\left(c_{n}-s\right)} \frac{L(n+1)}{L(n)} \cdot \frac{L(n+2)}{L(n+1)} \cdots \frac{L\left(n+c_{n}-s\right)}{L\left(n+c_{n}-s-1\right)} \\
& <C_{\alpha} p^{-\alpha\left(c_{n}-s\right)} \cdot\left(p^{\alpha / 2}\right)^{c_{n}-s} \\
& =C_{\alpha} p^{-\alpha\left(c_{n}-s\right) / 2} .
\end{aligned}
$$


If we take $M^{\prime} \geq M$ sufficiently large, then for any $n \geq M^{\prime}$ the last term in (9) is less than 3 , and thus the right-hand side of (8) is positive. Hence we can derive from (6) and (7) that

$$
\begin{aligned}
& P_{n, s} \\
& \quad \leq N t \\
& \leq C_{\alpha} p^{-\alpha\left(c_{n}-s\right)} \frac{L\left(n+c_{n}-s\right)}{L(n)} \\
& =C_{\alpha} p^{-\alpha\left(c_{n}-s\right)} \frac{L\left(n+c_{n}\right)}{L(n)} \cdot \frac{L\left(n+c_{n}-1\right)}{L\left(n+c_{n}\right)} \cdot \frac{L\left(n+c_{n}-2\right)}{L\left(n+c_{n}-1\right)} \cdots \frac{L\left(n+c_{n}-s\right)}{L\left(n+c_{n}-s+1\right)} \\
& <C_{\alpha} p^{-\alpha\left(c_{n}-s\right)} \frac{L\left(n+c_{n}\right)}{L(n)}\left(p^{\alpha / 2}\right)^{s} \\
& =C_{\alpha} p^{3 \alpha s / 2} \cdot p^{-\alpha c_{n}} \frac{L\left(n+c_{n}\right)}{L(n)}
\end{aligned}
$$

provided $n \geq M^{\prime}$. Accordingly, the assumption $\mathcal{S}<+\infty$ implies

$$
\sum_{n=M^{\prime}}^{\infty} P_{n, s} \leq C_{\alpha} p^{3 \alpha s / 2} \mathcal{S}<+\infty,
$$

and by Borel-Cantelli's lemma,

$$
P\left(\max _{1 \leq i \leq[N(n)]}\left|S_{i}\right|_{p} \geq p^{n+c_{n}-s} \text { for infinitely many } n\right)=0 .
$$

Hence

$$
\limsup _{n \rightarrow \infty}\left|p^{n+c_{n}} S_{[N(n)]}\right|_{p}=\limsup _{n \rightarrow \infty} p^{-n-c_{n}}\left|S_{[N(n)]}\right|_{p}<p^{-s}
$$

holds almost surely, and since $s$ is arbitrary, we conclude that $\lim _{\sup } \rightarrow \infty\left|p^{n+c_{n}} S_{[N(n)]}\right|_{p}=0$. Then it is clear that $p^{n+c_{n}} S_{[N(n)]}$ converges to 0 almost surely.

(ii) Since

$$
N(n)-N(n-1)=C_{\alpha} T\left(p^{n}\right)^{-1}\left(1-p^{-\alpha} \frac{L(n)}{L(n-1)}\right) \rightarrow+\infty
$$

as $n \rightarrow \infty$, we can assume $[N(n)]>[N(n-1)]$ for large $n$. Let us put $Q_{n, s}:=P\left(\left|S_{[N(n)]}-S_{[N(n-1)]}\right| p\right.$ $\geq p^{n+c_{n}+s}$ ) for natural numbers $n$ and $s$, then Lemma 3.1 yields

$$
\begin{aligned}
Q_{n, s} & =1-A_{n+c_{n}+s}([N(n)],[N(n-1)]) \\
& \geq \frac{1}{2}\left(1-\left(1-2 T\left(p^{n+c_{n}+s}\right)\right)^{[N(n)]-[N(n-1)]}\right) .
\end{aligned}
$$

Here for $0<t<1$ and a natural number $N$, we see

$$
\begin{aligned}
1- & (1-t)^{N} \\
= & -\sum_{k=1}^{N}\left(\begin{array}{l}
N \\
k
\end{array}\right)(-t)^{k} \\
& =\left\{\begin{array}{l}
N t-\frac{N(N-1)}{2} t^{2}+\sum_{j=2}^{\frac{N}{2}}\left(\left(\begin{array}{c}
N \\
2 j-1
\end{array}\right) t^{2 j-1}-\left(\begin{array}{c}
N \\
2 j
\end{array}\right) t^{2 j}\right), \quad \text { if } \quad N \text { is even, } \\
N t-\frac{N(N-1)}{2} t^{2}+\sum_{j=2}^{\frac{N-1}{2}}\left(\left(\begin{array}{c}
N \\
2 j-1
\end{array}\right) t^{2 j-1}-\left(\begin{array}{c}
N \\
2 j
\end{array}\right) t^{2 j}\right)+t^{N} \\
\quad \geq N t-\frac{N(N-1)}{2} t^{2}+\sum_{j=2}^{\frac{N-1}{2}}\left(\left(\begin{array}{c}
N \\
2 j-1
\end{array}\right) t^{2 j-1}-\left(\begin{array}{c}
N \\
2 j
\end{array}\right) t^{2 j}\right), \quad \text { if } \quad N \text { is odd, }
\end{array}\right.
\end{aligned}
$$


and the each term in these sums is estimated as

$$
\begin{aligned}
& \left(\begin{array}{c}
N \\
2 j-1
\end{array}\right) t^{2 j-1}-\left(\begin{array}{c}
N \\
2 j
\end{array}\right) t^{2 j} \\
& =\frac{N !}{(2 j-1) !(N-2 j+1) !} t^{2 j-1}\left(1-\frac{N-2 j+1}{2 j} t\right) \\
& \geq \frac{N !}{(2 j-1) !(N-2 j+1) !} t^{2 j-1}(1-N t) .
\end{aligned}
$$

Since $\frac{L(n+1)}{L(n)} \rightarrow 1$ and $T\left(p^{n}\right) \rightarrow 0$ as $n \rightarrow \infty$, there exists $M \geq 1$ such that $p^{-\alpha / 2}<\frac{L(n+1)}{L(n)}<p^{\alpha / 2}$ and $T\left(p^{n+c_{n}+s}\right)<\frac{1}{2}$ hold for all $n \geq M$. Put $N=[N(n)]-[N(n-1)]$ and $t=2 T\left(p^{n+c_{n}+s}\right)$, then for $n \geq M$ we have

$$
\begin{aligned}
N & \\
\leq & 2(N(n)-N(n-1)+1) T\left(p^{n+c_{n}+s}\right) \\
= & 2 C_{\alpha}\left(1-p^{-\alpha} \frac{L(n)}{L(n-1)}\right) p^{-\alpha\left(c_{n}+s\right)} \frac{L\left(n+c_{n}+s\right)}{L(n)}+2 T\left(p^{n+c_{n}+s}\right) \\
= & 2 C_{\alpha}\left(1-p^{-\alpha} \frac{L(n)}{L(n-1)}\right) p^{-\alpha\left(c_{n}+s\right)} \frac{L(n+1)}{L(n)} \cdot \frac{L(n+2)}{L(n+1)} \cdots \frac{L\left(n+c_{n}+s\right)}{L\left(n+c_{n}+s-1\right)} \\
& +2 T\left(p^{n+c_{n}+s}\right) \\
< & 2 C_{\alpha}\left(1-p^{-\alpha} \frac{L(n)}{L(n-1)}\right) p^{-\alpha\left(c_{n}+s\right)} \cdot\left(p^{\alpha / 2}\right)^{c_{n}+s}+2 T\left(p^{n+c_{n}+s}\right) \\
= & 2 C_{\alpha}\left(1-p^{-\alpha} \frac{L(n)}{L(n-1)}\right) p^{-\alpha\left(c_{n}+s\right) / 2}+2 T\left(p^{n+c_{n}+s}\right) .
\end{aligned}
$$

Since $\frac{L(n)}{L(n-1)} \rightarrow 1, p^{-\alpha\left(c_{n}+s\right) / 2} \rightarrow 0$, and $T\left(p^{n+c_{n}+s}\right) \rightarrow 0$ as $n \rightarrow \infty$, we can take $M^{\prime}>M$ such that the right-hand side of (12) is less than 1 for any $n \geq M^{\prime}$. Then for $n \geq M^{\prime}$ the right-hand side of (11) is positive, and hence $1-(1-t)^{N} \geq N t-\frac{N(N-1)}{2} t^{2}$. If $M^{\prime}$ is sufficiently large, we can assume $N(n)-N(n-1) \geq 2$, and then (10) leads to

$$
\begin{aligned}
Q_{n, s} \geq & \frac{1}{2}\left(N t-\frac{N(N-1)}{2} t^{2}\right) \\
> & \frac{1}{2} N t\left(1-\frac{N}{2} t\right) \\
> & \frac{1}{2} N t \cdot \frac{1}{2} \\
= & \frac{1}{2}([N(n)]-[N(n-1)]) T\left(p^{n+c_{n}+s}\right) \\
\geq & \frac{1}{4}(N(n)-N(n-1)) T\left(p^{n+c_{n}+s}\right) \\
= & \frac{1}{4} C_{\alpha}\left(1-p^{-\alpha} \frac{L(n)}{L(n-1)}\right) p^{-\alpha\left(c_{n}+s\right)} \frac{L\left(n+c_{n}+s\right)}{L(n)} \\
= & \frac{1}{4} C_{\alpha}\left(1-p^{-\alpha} \frac{L(n)}{L(n-1)}\right) p^{-\alpha\left(c_{n}+s\right)} \frac{L\left(n+c_{n}\right)}{L(n)} \\
& \times \frac{L\left(n+c_{n}+1\right)}{L\left(n+c_{n}\right)} \cdot \frac{L\left(n+c_{n}+2\right)}{L\left(n+c_{n}+1\right)} \cdots \frac{L\left(n+c_{n}+s\right)}{L\left(n+c_{n}+s-1\right)} \\
> & \frac{1}{4} C_{\alpha}\left(1-p^{-\alpha} \cdot p^{\alpha / 2}\right) p^{-\alpha\left(c_{n}+s\right)} \frac{L\left(n+c_{n}\right)}{L(n)} \cdot\left(p^{-\alpha / 2}\right)^{s} \\
= & \frac{1}{4} C_{\alpha}\left(1-p^{-\alpha / 2}\right) p^{-3 \alpha s / 2} p^{-\alpha c_{n}} \frac{L\left(n+c_{n}\right)}{L(n)} .
\end{aligned}
$$


Then the assumption $\mathcal{S}=+\infty$ implies

$$
\sum_{n=M^{\prime}}^{\infty} Q_{n, s} \geq \frac{1}{4} C_{\alpha}\left(1-p^{-\alpha / 2}\right) p^{-3 \alpha s / 2} \sum_{n=M^{\prime}}^{\infty} p^{-\alpha c_{n}} \frac{L\left(n+c_{n}\right)}{L(n)}=+\infty,
$$

and since the events $\left|S_{[N(n)]}-S_{[N(n-1)]}\right|_{p} \geq p^{n+c_{n}+s}$ are independent for $n=1,2, \ldots$, Borel's theorem shows

$$
P\left(\left|S_{[N(n)]}-S_{[N(n-1)]}\right|_{p} \geq p^{n+c_{n}+s} \text { for infinitely many } n\right)=1 .
$$

If we suppose $\left|S_{[N(n)]}(\omega)\right|_{p}<p^{n+c_{n}+s}$ for all large $n$, then by the ultra-metric property,

$$
\begin{aligned}
\left|S_{[N(n)]}(\omega)-S_{[N(n-1)]}(\omega)\right|_{p} & \leq \max \left\{\left|S_{[N(n)]}(\omega)\right|_{p},\left|S_{[N(n-1)]}(\omega)\right|_{p}\right\} \\
& <\max \left\{p^{n+c_{n}+s}, p^{n-1+c_{n-1}+s}\right\} \\
& =p^{n+c_{n}+s}
\end{aligned}
$$

holds for all large $n$. Whereas the probability of such an event is 0 according to (13), and consequently we have $\lim \sup _{n \rightarrow \infty}\left|p^{n+c_{n}} S_{[N(n)]}\right|_{p}=p^{-n-c_{n}}\left|S_{[N(n)]}\right|_{p} \geq p^{s}$ with probability 1. Letting $s \rightarrow \infty$, we obtain $\lim \sup _{n \rightarrow \infty}\left|p^{n+c_{n}} S_{[N(n)]}\right|_{p}=+\infty$ almost surely.

Following Lemma 3.3, we shall look for the critical order of the sequence $\left\{c_{n}\right\}$ over which the scaled sum $p^{n+c_{n}} S_{[N(n)]}$ converges to 0 almost surely. For the first try, let us examine positive powers of $n$.

Proposition 3.4 For any $\beta>0$ and $\varepsilon>0, c_{n}=\left[\beta n^{\varepsilon}\right]$ gives $p^{n+c_{n}} S_{[N(n)]} \rightarrow 0$ almost surely.

Proof According to the assumption $\frac{L(n+1)}{L(n)} \rightarrow 1$, take $M \geq 1$ such that $\frac{L(n+1)}{L(n)}<p^{\alpha / 2}$ holds for any $n \geq M$. For each natural number $l, c_{n}=\left[\beta n^{\varepsilon}\right]$ takes the value $l$ if and only if $\left(\frac{l}{\beta}\right)^{1 / \varepsilon} \leq n<\left(\frac{l+1}{\beta}\right)^{1 / \varepsilon}$, and therefore

$$
\begin{aligned}
& \sum_{n=M}^{\infty} p^{-\alpha c_{n}} \frac{L\left(n+c_{n}\right)}{L(n)} \\
& \leq \sum_{l=c_{M}}^{\infty} \sum_{\left(\frac{l}{\beta}\right)^{1 / \varepsilon} \leq n<\left(\frac{l+1}{\beta}\right)^{1 / \varepsilon}} p^{-\alpha l} \frac{L(n+1)}{L(n)} \cdot \frac{L(n+2)}{L(n+1)} \cdots \frac{L(n+l)}{L(n+l-1)} \\
& \leq \sum_{l=c_{M}}^{\infty}\left(\left(\frac{l+1}{\beta}\right)^{1 / \varepsilon}-\left(\frac{l}{\beta}\right)^{1 / \varepsilon}\right) p^{-\alpha l} \cdot\left(p^{\alpha / 2}\right)^{l} \\
& =\beta^{-1 / \varepsilon} \sum_{l=c_{M}}^{\infty}\left((l+1)^{1 / \varepsilon}-l^{1 / \varepsilon}\right) p^{-\alpha l / 2} \\
& \leq \beta^{-1 / \varepsilon} \sum_{l=c_{M}}^{\infty}(l+1)^{1 / \varepsilon} p^{-\alpha l / 2} .
\end{aligned}
$$

Here putting $d_{l}:=(l+1)^{1 / \varepsilon} p^{-\alpha l / 2}$, we have

$$
\frac{d_{l+1}}{d_{l}}=\left(\frac{l+2}{l+1}\right)^{1 / \varepsilon} p^{-\alpha / 2} \rightarrow p^{-\alpha / 2}<1
$$

as $l \rightarrow \infty$. Then by d'Alembert's ratio test the sum $\sum_{n=M}^{\infty} p^{-\alpha c_{n}} \frac{L\left(n+c_{n}\right)}{L(n)}$, and hence $\mathcal{S}$ is convergent. Thus the assertion follows by Lemma 3.3.

This proposition shows that any positive power of $n$ is too fast for a candidate of the critical order of $c_{n}$. The correct order is $c_{n} \sim \beta \log n$ for some $\beta>0$, as is given in Theorem 1.4. 


\section{Proofs of Theorems}

Proof (Theorem 1.4) (i) Put $\alpha \beta \log p=1+\delta, \delta>0$, then $p^{-\alpha c_{n}}<p^{-\alpha(\beta \log n-1)}=p^{\alpha} n^{-1-\delta}$. By the assumption $\frac{L(n+1)}{L(n)} \rightarrow 1$, we can take $M \geq 1$ such that $\frac{L(n+1)}{L(n)}<p^{\alpha \delta / 2(1+\delta)}$ holds for all $n \geq M$. Then for $n \geq M$ it follows that

$$
\begin{aligned}
\frac{L\left(n+c_{n}\right)}{L(n)} & =\frac{L(n+1)}{L(n)} \cdot \frac{L(n+2)}{L(n+1)} \cdots \frac{L\left(n+c_{n}\right)}{L\left(n+c_{n}-1\right)} \\
& <\left(p^{\alpha \delta / 2(1+\delta)}\right)^{c_{n}} \\
& \leq\left(p^{\alpha \delta / 2(1+\delta)}\right)^{\beta \log n} \\
& =n^{\delta / 2},
\end{aligned}
$$

and therefore

$$
\sum_{n=M}^{\infty} p^{-\alpha c_{n}} \frac{L\left(n+c_{n}\right)}{L(n)} \leq p^{\alpha} \sum_{n=M}^{\infty} n^{-1-\delta / 2}<+\infty .
$$

This implies $\mathcal{S}<+\infty$, and then the assertions follow by Lemma 3.3.

(ii) Put $\alpha \beta \log p=1-\delta, \delta>0$, then $p^{-\alpha c_{n}} \geq p^{-\alpha \beta \log n}=n^{-1+\delta}$. For some $M \geq 1$, we can suppose $\frac{L(n+1)}{L(n)}>p^{-\alpha \delta / 2(1-\delta)}$ for all $n \geq M$, and then

$$
\begin{aligned}
\frac{L\left(n+c_{n}\right)}{L(n)} & =\frac{L(n+1)}{L(n)} \cdot \frac{L(n+2)}{L(n+1)} \cdots \frac{L\left(n+c_{n}\right)}{L\left(n+c_{n}-1\right)} \\
& >\left(p^{-\alpha \delta / 2(1-\delta)}\right)^{c_{n}} \\
& \geq\left(p^{-\alpha \delta / 2(1-\delta)}\right)^{\beta \log n} \\
& =n^{-\delta / 2} .
\end{aligned}
$$

Hence we have

$$
\mathcal{S} \geq \sum_{n=M}^{\infty} p^{-\alpha c_{n}} \frac{L\left(n+c_{n}\right)}{L(n)} \geq \sum_{n=M}^{\infty} n^{-1+\delta / 2}=+\infty,
$$

and Lemma 3.3 implies $\lim \sup _{n \rightarrow \infty}\left|p^{n+c_{n}} S_{[N(n)]}\right|_{p}=+\infty$ a.s.

Our next interest is in what happens at the critical order $c_{n} \sim \frac{\log n}{\alpha \log p}$. Let us examine the simplest case where $L(n)$ is constant for $n \geq 1$.

Example 4.1 In case $c_{n}=\left[\frac{\log n}{\alpha \log p}\right]$ and $\frac{L(n+1)}{L(n)} \equiv 1$ for $n \geq 1$, we have

$$
\limsup _{n \rightarrow \infty}\left|p^{n+c_{n}} S_{[N(n)]}\right|_{p}=+\infty .
$$

Indeed, we see

$$
\mathcal{S}=\sum_{n=1}^{\infty} p^{-\alpha c_{n}} \geq \sum_{n=1}^{\infty} p^{-\alpha \frac{\log n}{\alpha \log p}}=\sum_{n=1}^{\infty} n^{-1}=+\infty .
$$

It cannot be concluded that the sum with the specific $\left\{c_{n}\right\}$ in the example diverges for all sequences $\{L(n)\}$. As Theorem 1.5 shows, at the critical order $c_{n}=\left[\frac{\log n}{\alpha \log p}\right]$, the convergence/divergence of $\lim \sup _{n \rightarrow \infty}\left|p^{n+c_{n}} S_{[N(n)]}\right|_{p}$ depends on the order of the convergence $\frac{L(n+1)}{L(n)} \rightarrow 1$. 
Proof (Theorem 1.5) Let us see

$$
\begin{aligned}
\frac{L\left(n+c_{n}\right)}{L(n)} & =\prod_{k=n}^{n+c_{n}-1} \frac{L(k+1)}{L(k)} \\
& =\prod_{k=n}^{n+c_{n}-1} e^{-\gamma \log \log k / \log k} \\
& =e^{-\gamma \sum_{k=n}^{n+c_{n}-1} \log \log k / \log k},
\end{aligned}
$$

and estimate $H_{n}:=\sum_{k=n}^{n+c_{n}-1} \frac{\log \log k}{\log k}$.

(i) Fix numbers $\rho$ and $\rho^{\prime}$ such that $\frac{\alpha \log p}{\gamma}<\rho^{\prime}<\rho<1$, and take a large integer $M$ so that $\log M \geq \frac{\alpha \log p}{1-\rho}$. Then for $n \geq M$, we have $(1-\rho) \frac{\log n}{\alpha \log p} \geq 1$ and then

$$
c_{n} \geq \frac{\log n}{\alpha \log p}-1 \geq \frac{\log n}{\alpha \log p}-(1-\rho) \frac{\log n}{\alpha \log p}=\frac{\rho \log n}{\alpha \log p} .
$$

Since $\frac{\log \log k}{\log k} \geq \frac{\log \log \left(n+c_{n}\right)}{\log \left(n+c_{n}\right)}$ holds for $k=n, n+1, \ldots, n+c_{n}-1$, it follows that

$$
H_{n} \geq c_{n} \frac{\log \log \left(n+c_{n}\right)}{\log \left(n+c_{n}\right)} \geq \frac{\rho \log n}{\alpha \log p} \cdot \frac{\log \log \left(n+c_{n}\right)}{\log \left(n+c_{n}\right)}
$$

Consider a function $f(t):=\frac{\log \log t}{\log t}$ for $t>e^{e}$, then $f(t)>0$ and its derivative $f^{\prime}(t)=-\frac{\log \log t-1}{t(\log t)^{2}}$ is negative and increasing for sufficiently large $t$. Therefore if $n$ is sufficiently large, we have

$$
\begin{aligned}
f\left(n+c_{n}\right) & \geq f(n)+f^{\prime}(n) c_{n} \\
& \geq f(n)-\frac{\log \log n-1}{n(\log n)^{2}} \cdot \frac{\log n}{\alpha \log p} \\
& =f(n)-\frac{1}{\alpha \log p} \cdot \frac{\log \log n-1}{n \log n},
\end{aligned}
$$

or equivalently

$$
\begin{aligned}
\frac{f\left(n+c_{n}\right)}{f(n)} & \geq 1-\frac{1}{\alpha \log p} \cdot \frac{\log \log n-1}{n \log n} \cdot \frac{1}{f(n)} \\
& =1-\frac{1}{\alpha \log p} \frac{\log \log n-1}{n \log \log n} .
\end{aligned}
$$

Since the right-hand side of (14) tends to 1 as $n \rightarrow \infty$, we can take $M^{\prime} \geq M$ such that $f\left(n+c_{n}\right) / f(n) \geq \frac{\alpha \log p}{\gamma \rho^{\prime}}$ for all $n \geq M^{\prime}$, and then

$$
H_{n} \geq \frac{\rho \log n}{\alpha \log p} \cdot \frac{\alpha \log p}{\gamma \rho^{\prime}} f(n)=\frac{\rho \log \log n}{\gamma \rho^{\prime}} .
$$

Put $\theta:=\frac{\rho}{\rho^{\prime}}>1$, then the above leads us to

$$
\frac{L\left(n+c_{n}\right)}{L(n)} \leq e^{-\gamma \cdot \rho \log \log n / \gamma \rho^{\prime}}=e^{-\theta \log \log n}=\frac{1}{(\log n)^{\theta}}
$$


for $n \geq M^{\prime}$, and consequently

$$
\begin{aligned}
\sum_{n=M^{\prime}}^{\infty} p^{-\alpha c_{n}} \frac{L\left(n+c_{n}\right)}{L(n)} & \leq \sum_{m=M^{\prime}}^{\infty} p^{-\alpha(\log n / \alpha \log p-1)} \cdot \frac{1}{(\log n)^{\theta}} \\
& =p^{\alpha} \sum_{n=M^{\prime}}^{\infty} \frac{1}{n(\log n)^{\theta}} \\
& \leq p^{\alpha} \int_{M^{\prime}-1}^{\infty} \frac{1}{t(\log t)^{\theta}} \mathrm{d} t<+\infty,
\end{aligned}
$$

since $\theta>1$. This implies $\mathcal{S}<+\infty$, and our assertion follows by Lemma 3.3.

(ii) Since $\frac{\log \log k}{\log k} \leq \frac{\log \log n}{\log n}$ for $k=n, n+1, \ldots, n+c_{n}-1$,

$$
H_{n} \leq c_{n} \frac{\log \log n}{\log n} \leq \frac{\log n}{\alpha \log p} \cdot \frac{\log \log n}{\log n}=\frac{\log \log n}{\alpha \log p} .
$$

Then

$$
\frac{L\left(n+c_{n}\right)}{L(n)} \geq e^{-\gamma \log \log n / \alpha \log p} \geq e^{-\log \log n}=\frac{1}{\log n},
$$

which leads to

$$
\begin{aligned}
\sum_{n=2}^{\infty} p^{-\alpha c_{n}} \frac{L\left(n+c_{n}\right)}{L(n)} & \geq \sum_{n=2}^{\infty} p^{-\alpha \cdot \log n / \alpha \log p} \frac{1}{\log n} \\
& =\sum_{n=2}^{\infty} \frac{1}{n \log n} \\
& \geq \int_{2}^{\infty} \frac{1}{t \log t} \mathrm{~d} t=+\infty .
\end{aligned}
$$

Thus we obtain $\mathcal{S}=+\infty$, and Lemma 3.3 gives $\lim \sup _{n \rightarrow \infty}\left|p^{n+c_{n}} S_{[N(n)]}\right|_{p}=+\infty$.

Acknowledgments This work was supported by Grant-in-Aid for Scientific Research (B) 21340020 .

Open Access This article is distributed under the terms of the Creative Commons Attribution License which permits any use, distribution, and reproduction in any medium, provided the original author(s) and the source are credited.

\section{References}

1. Gnedenko, B.V.; Kolmogorov, A.N.: Limit Distributions for Sums of Independent Random Variables. Addison-Wesley, London (1968)

2. Ito, K.: An Introduction to Probability Theory. Cambridge University Press, Cambridge (1986)

3. Petrov, V.V.: Limit Theorems of Probability Theory. Oxford Science Publications, Oxford (1995)

4. Yasuda, K.: On infinitely divisible distributions on locally compact Abelian groups. J. Theor. Probab. 13, 635-657 (2000) 